\title{
Does 5-alpha Reductase Inhibitor Use Improve The Efficacy of Intravesical Bacille Calmette-Guérin (BCG) for Non-muscle Invasive Bladder Cancer?
}

\author{
Mofarej Al-Hogbani, Sophie Gilbert, Michele Lodde, Yves Fradet and Paul Toren* \\ Department of Surgery, Faculty of Medicine, Centre Hospitalier Universitaire (CHU) de Québec Research \\ Center, Oncology Division, Université Laval, Québec, QC, Canada
}

Received 20 November 2019

Accepted 11 February 2020

\begin{abstract}
. patients with non-muscle invasive bladder cancer (NMIBC). was higher among 5-ARI users $(39.7 \%$ vs $17.9 \%, p=0.07)$.

Keywords: 5-alpha reductase inhibitor, bladder cancer, BCG, recurrence

*Correspondence to: Paul Toren, MD, PhD, FRCSC Assistant Professor, Université Laval, Research Scientist, Centre de Recherche du CHU de Québec-Université Laval and Centre de Recherche sur le Cancer, Université Laval, Uro-Oncologist, CHU de Québec-Université Laval, 10 McMahon, rm 0877 Québec, QC, Canada, G1R 3S1. Tel.: +1 4185254444 ext. 17064; Fax: +1 418 691 5562; E-mail: paul.toren@crchudequebec.ulaval.ca.
\end{abstract}

BACKGROUND: Prior research implicates the androgen receptor pathway as important in bladder cancer progression and recurrence. In particular, use of 5-alpha reductase inhibitors (5-ARIs) appears to improve bladder cancer outcomes. This study aims to determine if concomitant use of 5-ARIs with intravesical Bacille Calmette-Guérin (BCG) decreases recurrences in

MATERIALS \& METHODS: This retrospective analysis included male patients diagnosed with NMIBC who were treated with induction intravesical BCG at our institution from 2013 to 2018. Patients were excluded who received prior induction BCG. Recurrence and progression-free survival were estimated using the Kaplan-Meier method. Univariate and multivariate analyses were performed using Cox proportional hazards and Poisson events models.

RESULTS: We identified 206 male patients, of whom 39 received 5-ARIs and 167 did not. Most patients (72.8\%) received $>6$ instillations of BCG, with 20.4\% completing all induction and maintenance treatments during a median follow up of 3.31 years. There were no significant differences in recurrence or progression-free survival between 5-ARI users or non-users ( $59 \%$ vs $55.7 \% ; p=0.72$ and $97.4 \%$ vs $98.2 \% ; p=1.00$, respectively). Similarly, no differences in the number of recurrences between groups was observed $(p=0.78)$. However, the proportion of patients who completed all prescribed BCG installations

CONCLUSIONS: In contrast to prior reports, our study does not suggest that 5-ARI use decreases the recurrence rate in men receiving induction BCG for NMIBC. However, our results suggest 5-ARI use may improve patient tolerance to BCG.

\section{INTRODUCTION}

Despite major advancements in systemic therapies and utilization of multimodal approaches for muscle invasive bladder cancer (MIBC), non-muscle invasive bladder cancer (NMIBC) management remains relatively unchanged for decades, with BCG installation as a primary therapy which decreases risk of disease recurrence and progression [1]. 
Epidemiologic data strongly supports the idea that men are at an increased risk of developing bladder cancer compared to women, although women are more likely to present with advanced disease [2-4]. While environmental or lifestyle factors, such as industrial chemicals and cigarette smoke exposure may contribute to these differences, recent studies demonstrate that even after adjusting for these factors, sex-related differences in incidence persist [5]. Biologic differences related to sex hormones and sex hormone receptor activity remain a principal explanation [6]. In particular, androgens and the androgen receptor have been implicated in the embryologic origin and development of the bladder as well as bladder carcinogenesis in animal models $[6,7]$.

Pre-clinical models have suggested surgical castration may lower the incidence of bladder cancer induced by a chemical carcinogen in male mice. More recently, retrospective clinical studies have suggested that 5-alpha reductase inhibitors (5-ARIs) or androgen deprivation therapy may decrease the incidence and progression of bladder cancer [8, 9]. Notably, a study by Shiota et al. indicated that use of 5ARIs or other androgen suppressive medication may decrease the recurrence rate of NMIBC, with a HR of 0.36 [8].

To our knowledge, the influence of a 5-ARI inhibitor on the efficacy of intravesical instillations of BCG has not been studied. Intravesical BCG remains an integral part of NMIBC treatment for over 40 years [1]. Our objective was therefore to identify if the combination of BCG with 5-ARI use may suppress bladder cancer recurrence compared to BCG alone.

\section{MATERIALS \& METHODS}

\section{Patients}

This study was approved by the research ethics committee of the CHU de Québec-Université Laval (\#2018-3745). As it was a retrospective chart review, informed consent was not deemed necessary or applicable by the research ethics committee. A retrospective analysis was conducted to identify all male patients with NMIBC treated at Hôtel-Dieu de Québec Hospital between 2013 and 2018. Pharmacy records and medical charts were consulted to obtain BCG dosing and clinicopathologic information, respectively. We assessed for any androgen suppression therapy including the use of 5-ARI (dutasteride, finasteride), gonadotropin- releasing hormone $(\mathrm{GnRH})$ agonists and antagonists, or antiandrogens (eg bicalutamide). Medication use was assessed at time of BCG therapy, with the administration of 5-ARIs initiated at least 6 months prior to most recent transurethral resection of bladder tumor (TURBT) pre-BCG. Completeness of BCG maintenance therapy was classified as previous [10]: Group I, patients treated with less than 6 induction BCG instillations; Group II, patients who completed induction BCG but that had less than one year of maintenance BCG; and Group III, patients who completed induction BCG and maintenance for $\geq 1$ year.

All patients included underwent complete TURBT and were recommended for their first induction BCG treatment according to current guidelines for intermediate and high-risk NMIBC [1]. Patients with CIS only were also included. Regular patient follow-up included surveillance cystoscopy and urinary cytology every 3 months during the first 2 years and every 6 months thereafter. Seven patients were excluded from the study for incomplete follow-up (less than 2 years) or insufficient data on medication use.

Tumor recurrence was defined as pathologyconfirmed tumor and tumor progression was defined as an increase from low-grade to high grade pathology, or the presence of pT1 or pT2 for the first time.

\section{Study variables}

For each male patient with NMIBC included in this analysis, the following factors were recorded: demographic characteristic of each patient, intraoperative and postoperative parameters and androgen suppressive medication use according to prescription databases and medication profiles recorded by pharmacists during inpatient admission or day surgery.

\section{Statistical analyses}

Descriptive statistics were used for the rate of bladder cancer recurrence and progression, the number of TURBTs, time to recurrences, number of BCG instillations and histopathology details. Recurrence and progression-free survival rate curves were generated using the Kaplan-Meier method and were compared using the log-rank test. Differences among groups were considered as significant when $P<0.05$. Univariate and multivariate analyses of data were performed using the Cox proportional hazards model with stepwise forward selection. Poisson regression was used to estimate recurrence rate ratios and 
Table 1

Clinical and pathological features of patients included in study

\begin{tabular}{|c|c|c|c|c|c|}
\hline & \multicolumn{4}{|c|}{$5 \alpha$-reductase inhibitor $(\%)$} & \multirow[t]{2}{*}{$p$-value } \\
\hline & \multicolumn{2}{|c|}{ NO } & \multicolumn{2}{|c|}{ YES } & \\
\hline \multicolumn{6}{|l|}{ Smoking } \\
\hline Never & 71 & $(42.5)$ & 14 & $(35.9)$ & 0.22 \\
\hline Current & 30 & $(18.0)$ & 4 & $(10.3)$ & \\
\hline Former & 66 & $(39.5)$ & 21 & $(53.8)$ & \\
\hline \multicolumn{6}{|l|}{ T stage } \\
\hline $\mathrm{pTa}$ & 78 & $(45.8)$ & 18 & $(52.9)$ & 0.56 \\
\hline pT1 & 66 & $(54.2)$ & 16 & $(47.1)$ & \\
\hline \multicolumn{6}{|l|}{ Grade } \\
\hline High grade & 139 & 83.2 & 38 & $(97.4)$ & 0.03 \\
\hline Low grade & 28 & 16.8 & 1 & (2.6) & \\
\hline \multicolumn{6}{|l|}{ Multifocal } \\
\hline Not applicable & 35 & $(21.0)$ & 6 & $(15.4)$ & 0.67 \\
\hline Multiple & 101 & $(60.5)$ & 24 & $(61.5)$ & \\
\hline Single & 31 & $(18.6)$ & 9 & $(23.1)$ & \\
\hline \multicolumn{6}{|l|}{ Tumor size $(\mathrm{cm})$} \\
\hline$<3 \mathrm{~cm}$ & 106 & $(63.5)$ & 26 & $(66.7)$ & 0.73 \\
\hline$>3 \mathrm{~cm}$ & 26 & $(15.6)$ & 7 & $(17.9)$ & \\
\hline Not applicable & 35 & $(21.0)$ & 6 & (15.4) & \\
\hline \multicolumn{6}{|l|}{ CIS } \\
\hline Yes & 57 & $(34.1)$ & 13 & $(33.3)$ & 0.64 \\
\hline No & 105 & $(62.9)$ & 26 & $(66.7)$ & \\
\hline Unknown & 5 & $(3.0)$ & 0 & 0 & \\
\hline \multicolumn{6}{|l|}{ BCG intravesical group } \\
\hline Group I: less than 6 instillations of induction & 50 & $(29.9)$ & 6 & $(15.4)$ & 0.08 \\
\hline Group II: induction+less than 9 instillations of maintenance & 87 & $(52.1)$ & 21 & $(53.8)$ & \\
\hline Group III: 6 instillations of induction+9 instillations of consolidation & 30 & $(18.0)$ & 12 & $(30.8)$ & \\
\hline
\end{tabular}

tumor rate ratios adjusted for stage, grade, number of tumors, and primary tumor diameter. A given rate ratio provided a formal comparison of the rate among 5-ARI patients to the rate of other patients. All analyses were performed using Stata version 9.4.

\section{RESULTS}

A total of 206 males met the inclusion criteria, including 39 (18.9\%) 5-ARI users and 167 (81.1 $\%)$ non-users. Thirty-one $(79.5 \%)$ patients received dutasteride $0.5 \mathrm{mg}$ daily and $8(20.5 \%)$ patients finasteride $5 \mathrm{mg}$ daily. No patients received other androgen suppressive therapy. The median age of men in the entire patient cohort was 70 (interquartile range (IQR): 68.6-71.6). However, patients who received 5-ARI were generally older than those who did not receive the medications. Median age among 5-ARI recipients was 74 years, which was 6 years greater than the age of controls who did not receive 5-ARIs $(p=0.01)$. All other baseline characteristics between the two groups were comparable (Table 1). In the entire patient cohort, $121(58.7 \%)$ had a smoking history, 177 (86\%) had high-risk NMIBC, 55 (26.7\%) had second look TURBT and $150(72,8 \%)$ received BCG maintenance therapy (BCG). Median follow up was 3.31 (IQR 3.4-3.9) years, with only 6 patients dying or being lost to follow-up in the first year. Generally, there was no statistically significant difference in the proportion of all cause between 5ARI users and non-users. Notably, all patients in the 5-ARI cohort had high-risk disease on initial TUR $[3,16]$.

Overall, intravesical recurrence was observed during follow-up in $16(41 \%)$ of patients who received 5-ARIs and $74(44.3 \%)$ of patients who did not $(p=0.72)$. There was no statistical difference in 5year recurrence free survival between 5-ARI users versus non-users (59\% vs $55.7 \% ; p=0.72$ ). Cox proportional hazards models assessed characteristics associated with disease recurrence in our cohort (Table 2). On multivariate analysis, smoking (HR $1.75,95 \% \mathrm{CI}=1.09-2.83, p=0.02$ ) was associated with a higher risk of recurrence. Furthermore, a Poisson regression model evaluates the impact of 5-ARI use on the total number of recurrences, which was not significantly different between patients who received 5ARIs and those who did not when adjusted for other baseline risk factors. Disease progression was observed in only one (2.6\%) 5-ARI user and in three $(1.8 \%)$ non-users. 
Table 2

Associations of baseline characteristics, Bacillus Calmette-Guérin (BCG) treatment and 5- $\alpha$ reductase inhibitor use on recurrence-free survival

\begin{tabular}{|c|c|c|c|c|c|c|}
\hline & \multicolumn{3}{|c|}{ Univariate } & \multicolumn{3}{|c|}{ Multivariate } \\
\hline & HR & $(95 \% \mathrm{CI})$ & $\overline{p \text {-value }}$ & $\overline{\mathrm{HR}}$ & $(95 \% \mathrm{CI})$ & $\overline{p \text {-value }}$ \\
\hline Age & 1.02 & $0.98-1.02$ & 0.86 & 0.99 & $0.97-1.01$ & 0.79 \\
\hline \multicolumn{7}{|l|}{ Smoking history } \\
\hline No & 1 & & - & 1 & & - \\
\hline Yes (current + former) & 1.68 & $1.08-2.61$ & 0.02 & 1.75 & $1.09-2.83$ & 0.02 \\
\hline \multicolumn{7}{|l|}{ T stage } \\
\hline pTa & 1 & & - & 1 & & - \\
\hline pT1 & 1.13 & $073-1.76$ & 0.55 & 1.10 & $0.70-2.83$ & 0.66 \\
\hline \multicolumn{7}{|l|}{ Grade } \\
\hline Low grade & 1 & & - & 1 & & - \\
\hline High grade & 1.125 & $0.61-2.06$ & 0.70 & 1.16 & $0.60-2.23$ & 0.65 \\
\hline \multicolumn{7}{|l|}{ Multifocal } \\
\hline Single & 1.98 & $0.94-4.1$ & 0.07 & 2.05 & $0.91-1.61$ & 0.12 \\
\hline Multiple & 2.13 & $1.14-3.99$ & 0.017 & 2.29 & $0.80-6.15$ & 0.08 \\
\hline \multicolumn{7}{|l|}{ Tumor size $(\mathrm{cm})$} \\
\hline$<3 \mathrm{~cm}$ & 1.97 & $1.05-3.6$ & 0.03 & 0.83 & $0.46-1.4$ & 0.53 \\
\hline$>3 \mathrm{~cm}$ & 2.70 & $1.29-5.66$ & 0.008 & 1 & & - \\
\hline \multicolumn{7}{|l|}{ CIS } \\
\hline Yes & 0.78 & $0.18-3.31$ & 0.74 & 1.08 & $0.24-4.82$ & 0.91 \\
\hline No & 1.10 & $0.26-4.5$ & 0.89 & 1.32 & $0.31-5.63$ & 0.70 \\
\hline \multicolumn{7}{|l|}{ BCG treatment received group } \\
\hline$<1$ year therapy (Group $I+$ Group II) & 1 & & - & 1 & & - \\
\hline$>1$ year therapy (Group III) & 1.026 & $0.623-1.68$ & 0.92 & 1.08 & $0.62-1.87$ & 0.77 \\
\hline \multicolumn{7}{|l|}{$5-\alpha$ reductase inhibitor therapy } \\
\hline Non-users & 1 & & - & 1 & & - \\
\hline Users & 0.92 & $0.53-1.59$ & 0.77 & 1 & $0.55-1.79$ & 0.99 \\
\hline
\end{tabular}

A pre-planned subgroup analysis subsequently evaluated the effect of 5-ARI use according to the completeness of BCG therapy. There a lower prevalence of 5-ARI users among those receiving only induction BCG versus those who also received BCG maintenance (groups I vs II and III) which was above the convention for statistical significance $(10.7 \%$ vs $22 \%, p=0.066$ ). On Kaplan-Meier analysis, there was no significant differences in recurrence-free survival by 5-ARI use among the subgroups of BCG treatment received (Fig. 1). However, the proportion of patients who completed prescribed BCG installations was higher among 5-ARI users (Table 3). Finally, we examined the effect of 5-ARI on tumor recurrence at one year from induction BCG treatment, finding a non-significant decreased recurrence rate among 5-ARI users (33.3\% vs $58.3 \%$, $p=0.61)$.

\section{DISCUSSION}

The treatment landscape of bladder cancer continues to evolve. The clinical relevance of the AR and AR inhibition in bladder cancer remains unclear, but the accessibility and familiarity to urologists or AR-targeting medications present excellent potential for combination therapies. Intravesical BCG is the only treatment recognized to decrease both recurrence pathologic progression rates of NMIBC [1]. However, BCG therapy still has limitations: approximately $30 \%$ of patients treated with intravesical BCG failed to respond, and treatment may cause bladder and systemic toxicity which is responsible for treatment discontinuation. Moreover, recurrent BCG shortages in certain regions in the last several years have been problematic. Therefore, novel therapeutic strategies are needed to improve the effectiveness of BCG for NMIBC.

In preclinical models the androgen receptor (AR) has been implicated in the development and progression of bladder cancer [6, 7, 11]. More recently, several clinical studies have suggested that men who receive 5-ARIs may have better bladder cancer outcomes $[8,9,12,13]$. Shiota et al. demonstrated a reduced bladder cancer recurrence rates among patients who received 5-ARIs or androgen deprivation therapy in a cohort of 228 Japanese men [8]. Wu et al. similarly reported in a cohort of 274 men that the use of androgen suppression therapy was associated with a lower risk of bladder tumor recurrence [9]. In a population-based cohort of 10,720 Finnish men diagnosed with bladder cancer, those 

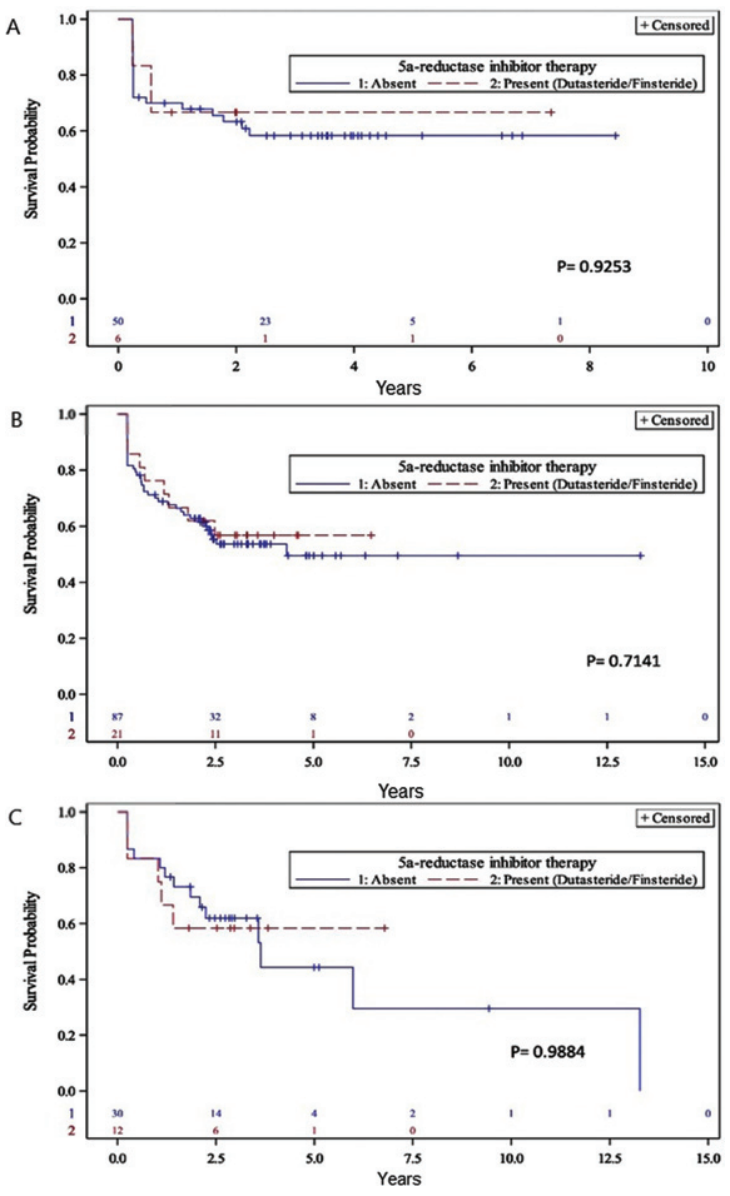

Fig. 1. Kaplan-Meier analysis of recurrence-free survival with and without 5-ARI stratified by amount of BCG received: (A) Group I $<6$ instillations (B) Group II $<1$ year maintenance (C) Group III at least 1 year maintenance.

who received 5-ARIs had a $25 \%$ reduction in disease specific survival compared to the control group who received $\alpha$-blockers [12]. Finally, an evaluation of AR expression in bladder tumors from patients who also received androgen deprivation therapy suggests that these beneficial effects may be due to an AR-mediated effect of the loss of androgens [14].

To our knowledge this is the first study to specifically assess whether 5-ARIs may impart a benefit in men with NIMBC receiving BCG therapy.
BCG therapy relies on the interaction between the bacillus and immunocompetent cells which stimulate the secretion of inflammatory cytokines such as interleukin (IL)-1 IL-2, IL-6 and interferon). Prior pre-clinical research has suggested that targeting AR signaling may enhance BCG immunotherapeutic effects via cytokine alterations [15].

Our results contrast previous reports which found a statistically significant association of androgen suppression therapy (AST) with disease progression for NIMBC $[8,9]$. AST in these studies was defined as 5-ARI, GnRH agonist or antagonist, or anti-androgen therapy; our study evaluated only 5ARIs. The time period in our study was shorter and more recent, though the follow-up time and number of patients is similar between studies. The most important difference appears that our study includes a more homogeneous cohort of patients, where only patients who received induction BCG were evaluated. Thus, the proportion of high-risk patients differences widely between our study and prior studies. With our inclusion criteria, $92 \%$ of patients in our study were high-risk, compared to $51 \%$ in the recent study by $\mathrm{Wu}$ [9]. Thus, $20 \%$ of high-risk patients in our study received 5-ARIs, compared to $11 \%$ in the study by $\mathrm{Wu}$ et al. No significant improvement in recurrences or progression with AST in their high-risk subgroup, with all NMIBC patients who progressed under AST initially showing high-risk disease [9]. With a highly homogeneous cohort, our study thus suggests 5-ARIs are ineffective to slow the progression of high-risk NMIBC.

The corollary is that the previously observed benefit of 5-ARIs may be most pronounced in patients with low grade, AR-expressing tumors. Prior immunohistochemistry studies have suggested that AR expression is more frequent in low grade tumors. Ide et al. conducted a meta-analysis to assess the expression of androgen receptor (AR) and estrogen receptors (ERs) in bladder cancer and found downregulation of AR expression in high-grade bladder tumors [16].

As a novel finding, we found fewer patients receiving 5-ARIs discontinued BCG treatments compared

Table 3

Number of Bacillus Calmette-Guérin (BCG) instillations received according to 5- $\alpha$ reductase inhibitor (5-ARI) use

\begin{tabular}{|c|c|c|c|c|c|c|c|}
\hline & \multicolumn{2}{|c|}{$\begin{array}{c}\text { Group I: } \\
<6 \text { instillations of } \\
\text { induction }\end{array}$} & \multicolumn{2}{|c|}{$\begin{array}{c}\text { Group II: } \\
<1 \text { year of instillations }\end{array}$} & \multicolumn{2}{|c|}{$\begin{array}{c}\text { Group III: } \\
>1 \text { year of instillations }\end{array}$} & \multirow[t]{2}{*}{$\overline{p \text {-value }}$} \\
\hline & $\mathrm{n} / \mathrm{N}$ & $\%$ & $\mathrm{n} / \mathrm{N}$ & $\%$ & $\mathrm{n} / \mathrm{N}$ & $\%$ & \\
\hline 5-ARI user & $6 / 56$ & 10.7 & $21 / 108$ & 19.4 & $12 / 42$ & 28.6 & 0.081 \\
\hline Non-user & $50 / 56$ & 89.3 & $87 / 108$ & 80.6 & $30 / 42$ & 71.4 & \\
\hline
\end{tabular}


to men who did not receive 5-ARIs. Reasons for discontinuation were presumed due to intolerance, though this information was not available from our chart review. Nonetheless, our results suggest it is worthwhile to further investigate whether 5-ARI use may improve the tolerability of men to BCG-induced lower urinary tract symptoms.

Our study has several strengths and limitations. Accurate assessment of 5-ARIs use was performed routinely at pre-operative pharmacist consultations. Further, the number of BCG treatments received was accurately recorded by our pharmacy department. Finally, we present a relatively large and homogeneous cohort of patients whose care aligned with established guidelines. Limitations include those inherent to single-institution retrospective studies and the lack of AR staining of tumor samples. Further, the proportion of patients who received 5-ARIs was low in our study, but comparable to prior studies.

In summary, our results suggest that 5-ARI use does not significantly decrease the risk of intravesical recurrence of NMIBC patients receiving BCG. In contrast to prior studies, this negative finding may be attributable to our population of NMIBC which was almost exclusively high-risk. Notably, we did however observe that discontinuation of BCG appears less frequent among patients who received 5-ARI compared to controls who did not. Further research is warranted to investigate this finding.

\section{ACKNOWLEDGMENTS}

We thank all the nurses in charge of our BCG unit for assistance in the care and follow up of bladder cancer patients. We are also grateful for the services of the statistical platform of the CHU de Québec Research Center, particularly Narcisse Singbo.

\section{FUNDING}

P. Toren is supported by a clinician-scientist award from Fonds de Recherche du Quebec - Sante (\#32774).

\section{AUTHOR CONTRIBUTIONS}

MA-H had full access to all the data in the study and takes responsibility for the integrity of the data and the accuracy of the data analysis; MA-H, ML, YF and PT contributed to the study concept and design; MA-H and $\mathrm{SG}$ contributed to the acquisition of data; $\mathrm{MA}-\mathrm{H}$ and PT contributed to the analysis and interpretation of data; MA-H drafted the manuscript; ML, YF and PT critically revised the manuscript for important intellectual content; MA-H and PT contributed to the statistical analysis; ML, YF and PT contributed to the administrative, technical or material support; ML, YF and PT contributed to the supervision.

\section{CONFLICT OF INTEREST}

YF reports research funding from Tersera, Astellas, IMV Inc; and personal fees as a consultant from Merck, Sanofi, Ferring, Amgen, Janssen, Astellas.

PT reports research funding from Bristol-MyersSquibb, Sanofi and Janssen; and personal fees as a consultant from Sanofi, Ferring, Tersera and Abbvie.

The other authors have no conflicts of interest to declare.

\section{REFERENCES}

[1] Babjuk M, et al. European Association of Urology Guidelines on Non-muscle-invasive Bladder Cancer (TaT1 and Carcinoma In Situ) - 2019 Update. Eur Urol. 2019;76:63957.

[2] Mungan NA, et al. Gender differences in stage-adjusted bladder cancer survival. Urology. 2000;55:876-80.

[3] Kluth LA, et al. Gender-specific differences in clinicopathologic outcomes following radical cystectomy: an international multi-institutional study of more than 8000 patients. Eur Urol. 2014;66:913-9.

[4] Uhlig A, et al. Gender-specific Differences in Recurrence of Non-muscle-invasive Bladder Cancer: A Systematic Review and Meta-analysis. Eur Urol Focus. 2018;4: 924-36.

[5] Wang S-C, et al. The gender difference and mortality-toincidence ratio relate to health care disparities in bladder cancer: National estimates from 33 countries. Sci Rep. 2017;7:4360.

[6] Li P, Chen J, Miyamoto H, Androgen Receptor Signaling in Bladder Cancer. Cancers (Basel). 2017;9.

[7] Miyamoto H, et al. Promotion of bladder cancer development and progression by androgen receptor signals. J Natl Cancer Inst. 2007;99:558-68.

[8] Shiota M, et al. Suppressed Recurrent Bladder Cancer after Androgen Suppression with Androgen Deprivation Therapy or $5 \alpha$-Reductase Inhibitor. J Urol. 2017;197: 308-13.

[9] Wu S-C, et al. Androgen Suppression Therapy Is Associated with Lower Recurrence of Non-muscleinvasive Bladder Cancer. European Urology Focus. doi:10.1016/j.euf.2019.04.021

[10] Alhogbani MM, Picard JA, Fassi-Fehri MH, Badet JL, Colombel CM, Prognostic impact of Bacillus CalmetteGuérin interruption at the time of induction and consolidation. Urol Ann. 2017;9:315-20.

[11] Johnson DT, et al. Conditional Expression of the Androgen Receptor Increases Susceptibility of Bladder Cancer in Mice. PLoS ONE 2016;11:e0148851. 
[12] Mäkelä VJ, Kotsar A, Tammela TLJ, Murtola TJ, Bladder Cancer Survival of Men Receiving $5 \alpha$-Reductase Inhibitors. J Urol. 2018. doi:10.1016/j.juro.2018.04.082

[13] McMartin C, et al. The use of 5-alpha reductase inhibitors prior to radical cystectomy - do they render high-grade bladder tumors less aggressive? Clin Genitourin Cancer. 2019.

[14] Izumi K, et al. Androgen deprivation therapy prevents bladder cancer recurrence. Oncotarget. 2014;5:12665-74.
[15] Shang Z, et al. Antiandrogen Therapy with Hydroxyflutamide or Androgen Receptor Degradation Enhancer ASC-J9 Enhances BCG Efficacy to Better Suppress Bladder Cancer Progression. Mol Cancer Ther. 2015;14: 2586-94.

[16] Ide H, Inoue S, Miyamoto H, Histopathological and prognostic significance of the expression of sex hormone receptors in bladder cancer: A meta-analysis of immunohistochemical studies. PLoS ONE. 2017;12:e0174746. 